\title{
Polymeric Biomaterial Based Hydrogels for Biomedical Applications
}

\author{
Nabanita Saha,* Aamarjargal Saarai, Niladri Roy, Takeshi Kitano, Petr Saha
}

Polymer Centre, Faculty of Technology, Tomas Bata University in Zlin, Zlin, Czech Republic.

Email: nabanita@ft.utb.cz,nabanitas@yahoo.com

Received October $2^{\text {nd }}, 2010$; revised December $15^{\text {th }}, 2010$; accepted December $20^{\text {th }}, 2010$.

\begin{abstract}
This paper focuses on the significant properties of hydrogels prepared with polymeric biomaterials: solely biopolymers (gelatin $(G)$ and sodium alginate $(S A)$ as base polymer) or in combination with synthetic and bio polymers (polyvinylpyrrolidone (PVP) and carboxymethylcellulose (CMC)) for biomedical application. Four kinds of hydrogels: G/SA, G/SA/SB (without and with seabuckthron oil (SB)) and PVP/CMC, PVP/CMC/BA (without and with boric acid (BA)) which are different from each other concerning shape, size, color, texture and properties point of view were achieved. $G / S A$ and G/SA/SB hydrogels vary from pale yellow to orange and a little rubber like having 42-48 \% moisture. On the other hand, PVP/CMC and PVP/CMC/BA hydrogels are transparent and soft gel like containing about 90-95\% moisture. Both G/SA and PVP/CMC hydrogels show similar trend of viscoelastic behaviour within whole range of measured

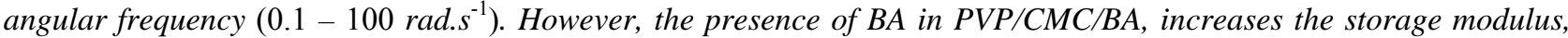
loss modulus and complex viscosity of hydrogel, and the presence of $S B$ in G/SA/SB demonstrates the decrease all of these values. G/SA based hydrogel possesses natural antimicrobial property whereas PVP/CMC based hydrogel needs to incorporate antimicrobial agent to comprise antimicrobial property within the hydrogel. G/SA hydrogels show water absorption capacity until 90 min whereas PVP/CMC hydrogels are able to absorb water steadily till 240 min. Finally, it can be mentioned that all four hydrogels: G/SA, G/SA/SB, PVP/CMC, PVP/CMC/BA which meet the basic requirements of hydrogel dressings, could be recommended as dressing materials for healing of burn or cut wound as well as a tool for transdermal drug delivery.
\end{abstract}

Keywords: Antimicrobial Property, Hydrogel, Polymeric Biomaterial, Transdermal Drug Delivery, Wound Dressings.

\section{Introduction}

A biomaterial is a synthetic material used to replace part of a living system or to function in intimate contact with living tissue [1]. The word "biomaterial" is generally used to recognize materials for biomedical applications. Biomaterials save lives, relieve suffering and improve the quality of life for a large number of patients every year. According to the use of materials in the body, biomaterials are classified into four groups: polymers, metals, ceramics and composites $[2,3]$.

Polymeric biomaterials (PB) are polysaccharides (starch, cellulose, chitin, alginate, hyaluronate etc.) or proteins (collagens, gelatins, caseins, albumins) and / or synthetic and biodegradable polymers (Polyvinyl alcohol (PVA), Polyvinylpyrrolidone (PVP), Polyetheleneglycol (PEG), Polylactic acid (PLA), Polyhydroxy acid (PHA) etc.). Currently, applications of polymeric biomaterials are promising for drug delivery, tissue engineering, bio- medical sensing, skin grafting, medical adhesives and textiles etc. It also covers targeted drug delivery to the nervous system, gastrointestinal tract, and kidneys etc. PB also includes the modern textile-based biomaterials for surgical applications; novel techniques in biomimetic polymer preparation; contemporary uses for polymers in dental and maxillofacial surgery; and many more [4]. From a practical perspective, medical applications of polymers fall into three broad categories: (i) extracorporeal uses (catheters, tubing, and fluid lines; dialysis membranes/artificial kidney; ocular devices; wound dressings and artificial skin), (ii) permanently implanted devices (sensory devices; cardiovascular devices; orthopedic devices; dental devices), and (iii) temporary implants (degradable sutures; implantable drug delivery systems; polymeric scaffolds for cell or tissue transplants; temporary vascular grafts and arterial stents ; temporary small bone fixation devices, transdermal drug delivery) $[5,6]$. 
Among all these prospective applications of $\mathrm{PB}$ in biomedical applications, concentration has given for the development of polymeric hydrogels as wound dressing cum wound healing material considering its application for extracorporeal purposes. Hydrogels are super absorbent polymeric materials which have significant roles in health care especially for wound treatment / protection. This might be due to their hydrophillicity, biocompatibility, non-toxicity, and biodegradability. Hydrogel possesses many remarkable properties such as immediate pain control effect, easy replacement, transparency, barrier against bacteria, good adhesion, easy handling, oxygen permeability, control of drug dosage, absorption, and prevention of loss of body fluids [7-14]. From healthcare points of view, hydrogel dressings have become a very interesting field of research for the development of a user friendly medical device for mankind. Numerous research studies prove that a moist wound environment is best for wounds to heal [15-19]. Two kinds of hydrogel based on PB have been developed, investigated in our laboratory and reported elsewhere $[8,9,12,13]$. The properties of two types of hydrogels (the one is prepared solely with biopolymers and the other is prepared in combination with biopolymer and synthetic polymer) were compared and mentioned in this paper.

\section{Experimental}

Polymeric (biopolymer) biomaterial based hydrogels: the hydrogels were prepared with biopolymers like gelatin $(\mathrm{G})$ and sodium alginate (SA) and without and with $0.5 \mathrm{ml}$ seabuckthorn oil (SB), as a skin care plus wound healing agent. Accordingly, the new hydrogels are named as $\mathrm{G} / \mathrm{SA}$ and G/SA/SB hydrogels. These hydrogels were prepared by applying physical stimulation technique; under constant stirring at $80^{\circ} \mathrm{C}$ for 5 min using ingredients (gelatin 5; sodium alginate 5; PEG 2; glycerin 2; $\mathrm{NaCl} 0.2$ $\mathrm{gm} / 20 \mathrm{ml}$ water)[8,9]. The semi solid polymeric mass (pseudo gel) was then placed in a grooved $(25 \mathrm{~mm}$ diameter and $2 \mathrm{~mm}$ thickness) acrylic plate and allowed it to become solid at room temperature.

Polymeric (synthetic and biopolymer) biomaterial based hydrogels: The other types of hydrogels were prepared with polyvinylpyrrolidone (PVP, synthetic polymer) and carboxymethylcellulose (CMC, biopolymer) incurporation of without and with $3 \%$ boric acid (BA), as an antimicrobial agent. Accordingly, the new hydrogels are named as PVP/CMC and PVP/CMC/BA hydrogels. These hydrogels were prepared under controlled pressure, moist heat and time (i.e. $15 \mathrm{lb}, 120^{\circ} \mathrm{C}$ and $15 \mathrm{~min}$ ) using ingredients (PVP/CMC 2:8; PEG 1; glycerin 1; agar $1 \mathrm{gm} / 100$ $\mathrm{ml}$ water).The liquid polymeric mass $(20 \mathrm{ml})$ was then transmitted in a Petri plate ( $80 \mathrm{~mm}$ diameter) and allowed to be cooled at room temperature to develop hydrogel (pseudo gel) $[12,13,20,21]$.

The essential and significant properties [physical (moisture content and morphology), viscoelastic, antimicrobial and swelling] of hydrogels are characterized and described below:

Moisture content of the hydrogels was measured gravimetrically by using the following equation (1).

$$
\left(M_{n}\right) \%=\left[\left(W_{w}-W_{d}\right) / W_{w}\right] \times 100
$$

Where, $M_{n}=$ moisture content (\%) of material $\mathrm{n}, W_{W}=$ wet weight of the sample, and $W_{d}=$ weight of the sample after drying at room temperature till they assumed constant weight [22]. Three replicates were taken and the average value of moisture content was determined.

The physical appearance (diameter and thickness) of hydrogels were measured using vernier scale and the interior morphologies were evaluated by scanning electron microscopy (SEM) analysis (VEGA II LMU (TESCAN)) operating in the high vacuum / secondary electron imaging mode at an accelerating voltage of 5-20 $\mathrm{kV})$. The freeze dried samples of before dry samples of hydrogels were analyzed [12]. The hydrogels were frozen under $-81^{\circ} \mathrm{C}$ for 72 hours and then lyophilized (ALPHA 1-4 LSC, Labicom s.r.o, Czech Republic) for 24 hours. Thereafter, the samples were sputter coated with a thin layer of palladium / gold alloy to improve the surface conductivity and tilted $30^{\circ}$ for better observation. The surface views as well as the cross-sectional views of the hydrogels were taken at magnification of $100 \mathrm{x}-10$ $\mathrm{kx}$.

The dynamic viscoelastic behavior of hydrogel samples were investigated by using a parallel plate rheometer (ARES; Rheometrics Scientific, USA) testing machine with an "RSI Orchestrator" software package. A $25 \mathrm{~mm}$ diameter parallel plate measuring geometry, with a gap of about 2-3 mm was used for the measurements under small strain amplitude $(1 \%)$ to maintain the measurements within the linear viscoelastic region (LVER). Dynamic frequency sweep tests were carried out at $28^{\circ} \mathrm{C}$ to observe the storage modulus (G') and loss modulus (G') as a function of a wide range of angular frequencies $(\omega$ : $0.1-100 \mathrm{rad} / \mathrm{s}$ ) [12,13]. In each case, three samples from the same hydrogel samples were measured.

The antimicrobial properties of hydrogels: G/SA, $\mathrm{G} / \mathrm{SA} / \mathrm{SB}$ and $\mathrm{PVP} / \mathrm{CMC}$, PVP/CMC/BA were investigated by agar diffusion method [13,23-25] where their antibacterial efficiency were examined on the basis of the dimension of inhibition zone generated in presence of Staphylococcus sp (bacteria) and Candida sp (fungus). The antimicrobial assay was conducted using sterile Nutrient Agar (2\%) medium for bacterial strain and Czapex Dox Agar (2\%) medium for fungal strain respectively. The testing plates were then incubated in a temperature 
controlled incubator at $37^{\circ} \mathrm{C}$ for Staphylococcus sp (bacteria) and at $30^{\circ} \mathrm{C}$ for Candida sp (fungus) to observe the effectivity of the hydrogels to inhibit the growth of microbes respectively.

The degree of swelling can be described as water absorptivity of the hydrogel. In case of G/SA and G/SA/SB, whole dry hydrogel samples were considered and for $\mathrm{PVP} / \mathrm{CMC}$ and PVP/CMC/BA, a small piece $\left(1 \mathrm{~cm}^{2}\right)$ from the dry films were weighed and immersed in distilled water at room temperature until reached to equilibrium state. After the specified time intervals, the water on the swollen gels was wiped off with tissue paper, and the weight of specimens was determined. The degree of swelling corresponds to the water absorptivity of the material, which is defined by equation 2 where, $W_{s}$ and $W_{d}$ are weights of swollen gel and dried gel, respectively [12,13, 26-28].

$$
\text { Absorption }(\%)=\left(W_{s}-W_{d}\right) / W_{d} \times 100
$$

\section{Results and Discussion}

Based on PB finally, four kinds of hydrogels i.e. G/SA, $\mathrm{G} / \mathrm{SA} / \mathrm{SB}$ and PVP/CMC, PVP/CMC/BA were achieved, which are actually different (shape, size, color, texture and property) from each other. The images of the above mentioned hydrogels are shown in Figure 1 and Figure 2, respectively.

The G/SA is pale yellow and rubber like during before dry state and then turned into hard, ivory colored at dry condition. Similarly, G/SA/SB is orange in color and rubber like turns into light yellow while dried (Figure 1). The observable orange color of $\mathrm{G} / \mathrm{SA} / \mathrm{SB}$ hydrogel is mainly due to the presence of SB oil in hydrogel.

The PVP/CMC and PVP/CMC/BA hydrogels are off white, transparent and soft gel like, moreover, no noticeable differences observed between the PB (synthetic and biopolymer) hydrogel at before dry state. But, after drying at
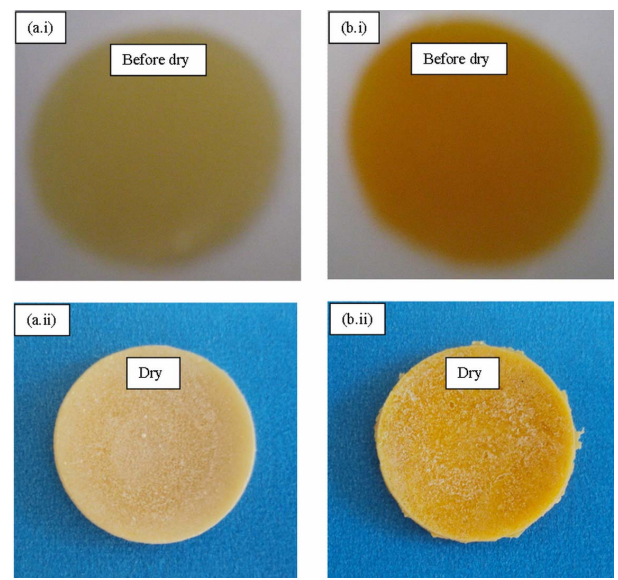

Figure 1. Optical images of hydrogels: G/SA (a.i) before dry (a.ii) dry and G/SA/SB (b.i) before dry (b.ii) dry.
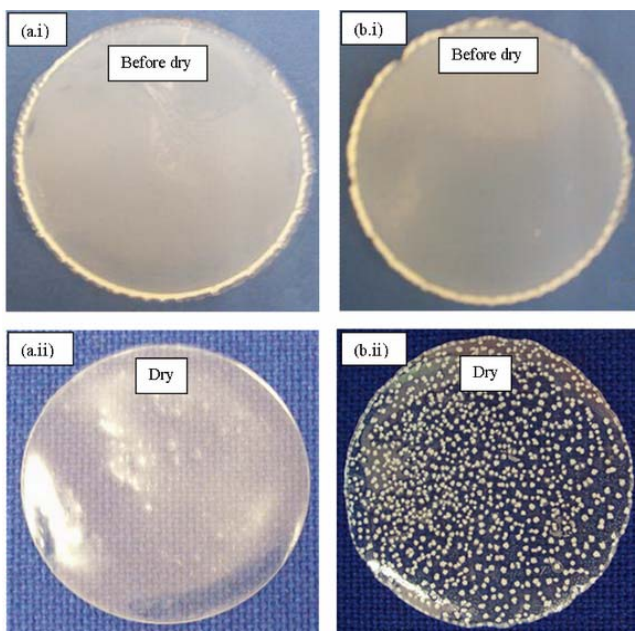

Figure 2. Optical images of hydrogels: PVP/CMC (a.i) before dry (a.ii) dry and PVP/CMC/BA (b.i) before dry (b.ii) dry.

room temperature, a remarkable difference is observed among these two hydrogels i.e. presence of BA is visible on the surface (in the form of white dots) of dry PVP/CMC/BA hydrogel (Figure 2).

While considering the physical appearance of PB hydrogels, it can be noticed that the G/SA and G/SA/SB decrease both in diameter and thickness after drying at room temperature. In each case of biopolymer based hydrogel the diameter is decreased from $25 \mathrm{~mm}$ to $20 \mathrm{~mm}$, thickness reduces from 2.437 to $1.869(\mathrm{G} / \mathrm{SA})$ and 2.245 to $1.865(\mathrm{G} / \mathrm{SA} / \mathrm{SB})$, respectively. Thus, it is assumed that due to the presence of SB oil, the G/SA/SB contains less water than G/SA. The moisture content of biopolymer based hydrogel varies between $42-48 \%$ (Table 1). Concerning the size, the PB (synthetic and biopolymer) hydrogel does not show any difference in diameter after drying at room temperature but they exhibit visible differences in thickness as they hold a large quantity of water (approximately 90-95\%) as shown in Table 2.

The surface topography and cross-sectional structure of the freeze dried samples of G/SA, G/SA/SB and $\mathrm{PVP} / \mathrm{CMC}, \mathrm{PVP} / \mathrm{CMC} / \mathrm{BA}$ hydrogels were examined by scanning electronic microscopy (SEM) and depicted in Figures 3 and 4, respectively. It can be seen that highly porous, flake like structure has been developed within G/SA and G/SA/SB hydrogels (Figure 3) without using

Table 1. Physical properties of polymeric (biopolymer) biomaterial based hydrogels.

\begin{tabular}{|c|c|c|c|c|c|}
\hline \multirow{2}{*}{$\begin{array}{l}\text { Sample } \\
\text { index }\end{array}$} & \multicolumn{2}{|c|}{ Diameter (mm) } & \multicolumn{2}{|c|}{ Thickness (mm) } & \multirow{2}{*}{$\begin{array}{l}\text { Moisture } \\
\text { content } \\
(\%)\end{array}$} \\
\hline & $\begin{array}{c}\text { Before } \\
\text { dry }\end{array}$ & Dry & $\begin{array}{c}\text { Before } \\
\text { dry }\end{array}$ & Dry & \\
\hline $\mathrm{G} / \mathrm{SA}$ & 25 & 20 & 2.437 & 1.869 & 48.4 \\
\hline $\mathrm{G} / \mathrm{SA} / \mathrm{SB}$ & 25 & 20 & 2.245 & 1.865 & 41.9 \\
\hline
\end{tabular}


Table 2. Physical properties of polymeric (bio and synthetic) biomaterial based hydrogel.

\begin{tabular}{|c|c|c|c|c|c|}
\hline \multirow{2}{*}{$\begin{array}{l}\text { Sample } \\
\text { index }\end{array}$} & \multicolumn{2}{|c|}{ Diameter mm) } & \multicolumn{2}{|c|}{ Thickness (mm) } & \multirow{2}{*}{$\begin{array}{c}\text { Moisture } \\
\text { content } \\
(\%)\end{array}$} \\
\hline & $\begin{array}{c}\text { Before } \\
\text { dry }\end{array}$ & Dry & $\begin{array}{c}\text { Before } \\
\text { dry }\end{array}$ & Dry & \\
\hline $\mathrm{PVP} / \mathrm{CMC}$ & 70 & 70 & 2.20 & 0.120 & 94.55 \\
\hline $\mathrm{PVP} / \mathrm{CMC} / \mathrm{BA}$ & 70 & 70 & 2.25 & 0.412 & 90.60 \\
\hline
\end{tabular}

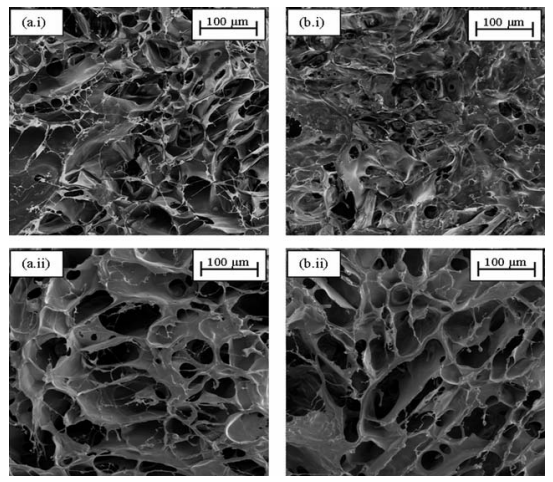

Figure 3. SEM images of hydrogels: G/SA (a.i) surface (a.ii) cross section and G/SA/SB (b.i) surface (b.ii) cross section.
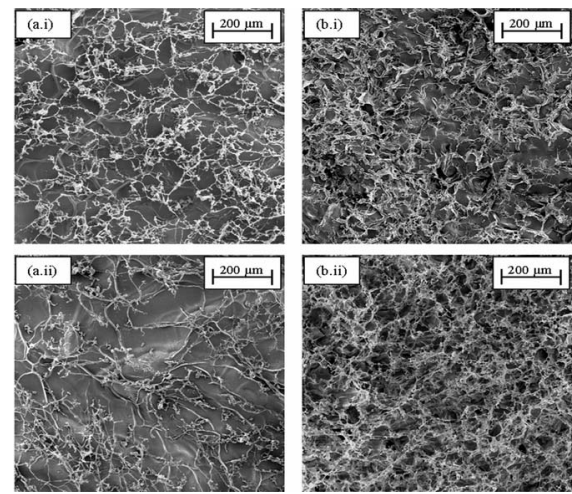

Figure 4. SEM images of hydrogels: PVP/CMC (a.i) surface (a.ii) cross section and PVP/CMC/BA (b.i) surface (b.ii) cross section.

any crosslinking agents. Only the influence of physical agents (temperature and stirring) stimulates to form the pseudo gel of G and SA. Presence of SB oil does not persuade much for enhancement of crosslinking network within the PB (biopolymer) based hydrogel. In addition, when comparing the internal structure of $\mathrm{PVP} / \mathrm{CMC}$ and $\mathrm{PVP} / \mathrm{CMC} / \mathrm{BA}$ hydrogels, PVP/CMC/BA exhibits more dense, sponge like crosslinking structure. The incorporation of $\mathrm{BA}$ in $\mathrm{PVP} / \mathrm{CMC}$ hydrogel increased the entanglement of the hydrogel network (Figure 4).

The viscoelastic behaviour of hydrogel is essential to study on application point of view as surface of the human body is uneven. It can be seen from the Figures 5 and 6 that both the hydrogels (G/SA and PVP/CMC) show normal gel properties i.e. storage modulus $\left(G^{\prime}\right)$ is

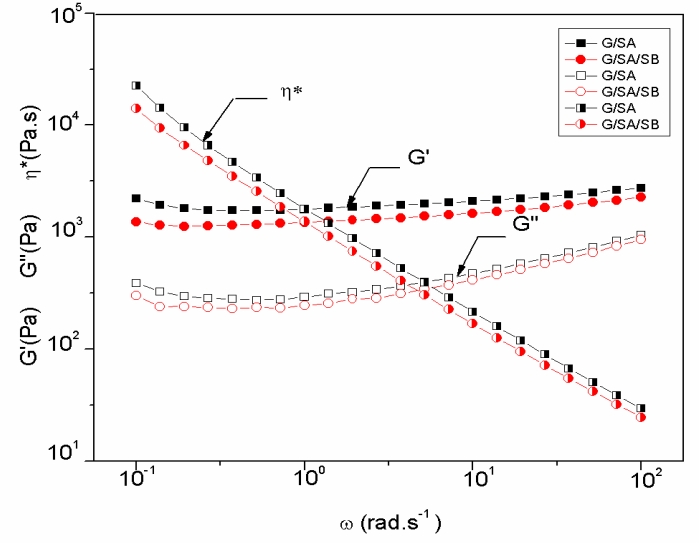

Figure 5. The viscoelastic behavior of hydrogels: G/SA and G/SA/SB.

higher than the loss modulus (G"). It can also be seen that both $\mathrm{G} / \mathrm{SA}$ and PVP/CMC hydrogels show similar trend of viscoelastic behaviour within whole range of angular frequency $\left(0.1-100 \mathrm{rad}_{\mathrm{s}} \mathrm{s}^{-1}\right)$ but their starting values (storage or elastic modulus, loss or viscous modulus and complex viscosity) are different. All these values are little reduced in $\mathrm{G} / \mathrm{SA} / \mathrm{SB}$ hydrogel due to the presence of seabuckthorn oil (SB) whereas in case of $\mathrm{PVP} / \mathrm{CMC} / \mathrm{BA}$ hydrogel it shows just an opposite behaviour. Presence of boric acid (BA) increased storage modulus, loss modulus even complex viscosity of hydrogel. It proves that $\mathrm{BA}$ helps to improve the cross linking net work between PVP and CMC. This effect of BA (the improvement of cross linking net work) is able to be observed in the SEM image shown in Figures $4 \mathbf{b}$ (i) and (ii).

The efficiency of antimicrobial properties of hydrogel is necessary to be determined before recommendation of its application for health care purposes as the presence of pathogenic microbes like bacteria (Staphylococcus $s p$ ) and fungi (Candida $s p$ ) are quite natural on wound infection, wound burn or fresh wound of skin. It can be seen from Figure 7 that both G/SA and G/SA/SB exhibit the antimicrobial effect in presence of both bacteria and fungi which is natural in origin. This may be due to the presence of sodium alginate which has antimicrobial property itself. Seabuckthorn oil (SB) does not play any role on this aspect, whereas BA (3\%) along with PVP/CMC hydrogel shows significant influence to achieve antimicrobial property as shown in Figure 8.

Water or fluid absorption capacity of the hydrogel is one of the important parameters from wound dressing point of view. When injury occurs on skin surface usually blood comes out which contains about $90 \%$ of water. Thus, prior to recommend about hydrogel for biomedical application it is prudent to work out the water uptake capacity of hydrogel. It can be seen from Figures $\mathbf{9}$ and $\mathbf{1 0}$ 


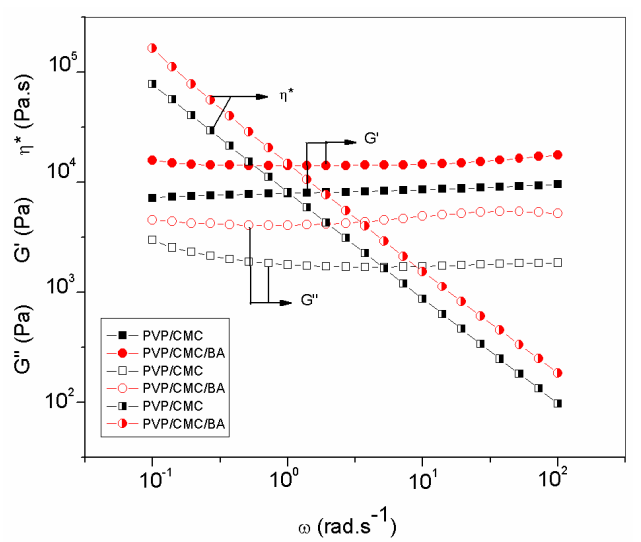

Figure 6. The viscoelastic behavior of hydrogels: PVP/CMC and PVP/CMC/BA.

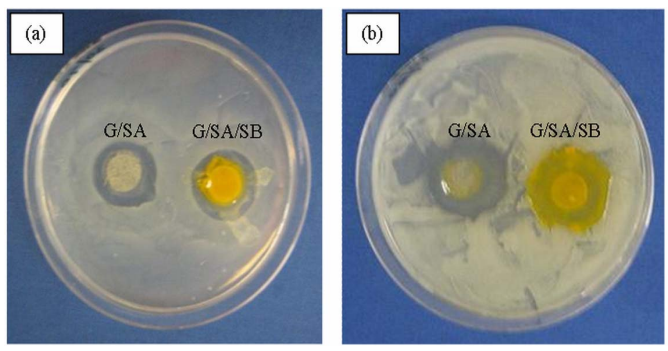

Figure 7. Images of antimicrobial properties of hydrogels: G/SA and G/SA/SB (a) Staphylococcus sp. (b) Candida sp.
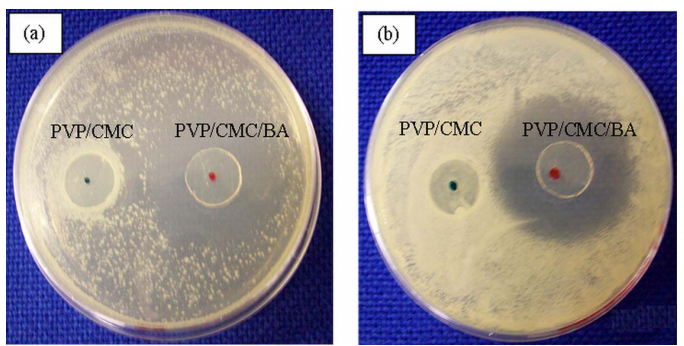

Figure 8. Images of antimicrobial properties of hydrogels: PVP/CMC and PVP/CMC/BA (a) Staphylococcus sp. (b) Candida sp.

that all hydrogels have reasonable water uptake capacity up to a long duration. G/SA hydrogels can absorb water until $90 \mathrm{~min}$ whereas PVP/CMC hydrogel can absorb water steadily till $240 \mathrm{~min}$. Further, it is recognized that the presence of seabuckthorn oil (SB) and boric acid (BA) for each hydrogel reduces the water uptake quantity. In the case of SB, the difference of water uptake quantity is not so significant however in the case of BA, it is very high.

\section{Conclusions}

All four kinds of hydrogels (G/SA \& G/SA/SB and $\mathrm{PVP} / \mathrm{CMC} \& \mathrm{PVP} / \mathrm{CMC} / \mathrm{BA}$ ) meet the basic requirements of existing dressing materials usually used for health care.

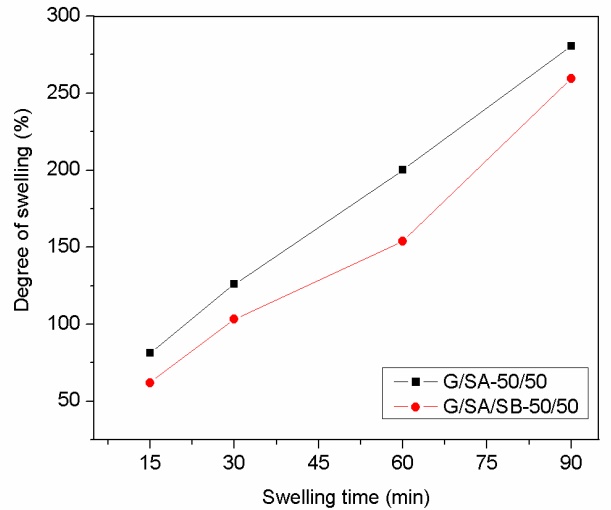

Figure 9. Swelling behavior of hydrogels: G/SA and G/SA/SB.

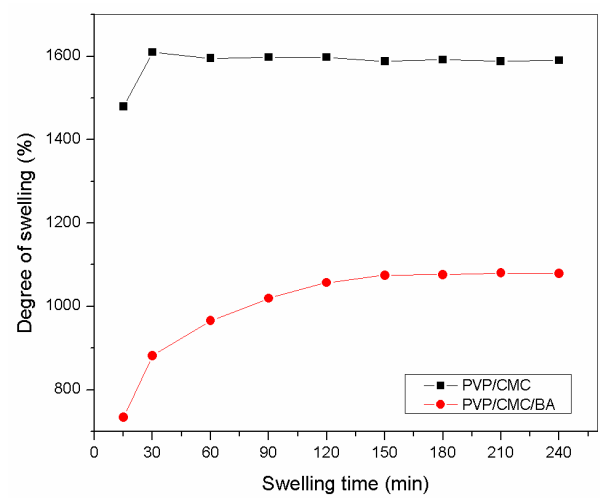

Figure 10. Swelling behavior of hydrogels: PVP/CMC and PVP/CMC/BA.

Thus, these hydrogels may be possible to use for health care purpose like: transdermal drug delivery, wound dressing, etc. It can be suggested that $\mathrm{G} / \mathrm{SA}$ and $\mathrm{G} / \mathrm{SA} / \mathrm{SB}$ hydrogels will be pretty good for wound healing cum wound protection and $\mathrm{PVP} / \mathrm{CMC}$ and $\mathrm{PVP} / \mathrm{CMC} / \mathrm{BA}$ hydrogels could be excellent for wound dressing cum wound healing purposes. Further, it can be pointed out that these hydrogels could be stored in dry form and can be used as and when required which is an additional advantage of PB based hydrogels. Moreover, these hydrogels could be recommended for their use as a medical device for transdermal drug delivery purposes as well.

\section{Acknowledgements}

The authors are thankful to the Ministry of Education, Youth and Sports of the Czech Republic (MSM 70088352101) for financial support.

\section{REFERENCES}

[1] B. D. Ratner, D. Hoffman, F. J. Schoen and J. E. Lemons, "Biomaterial Science; An Introduction to Materials in Medicine," Academic press, San Diego, 2004. 
[2] D. Shi, "Biomaterials and Tissue Engineering," SpringerVerlag, Berlin Heidelberg, 2004.

[3] B. Menaa, F. Menaa, C. Aiolfi-Guimaraes and O. Sharts, "Silica-based nanoporous sol-gel glasses: from bioencapsulation to protein folding studies," International Journal of Nanotechnology, Vol. 7, No. 1, 2010, pp. 1-45. doi:10.1504/IJNT.2010.029546

[4] S. C. Anand, J. F. Kennedy, M. Miraftab and S. Rajendran, "Medical textiles and biomaterials for healthcare," Woodhead Publishing Ltd, Cambridge, 2006.

[5] S. Dumitriu, "Polymeric Biomaterials, 2nd Ed," Marcel Dekker publisher, New York, 2002.

[6] I. Zhang, K. K. Shung and D. A. Edwards, "Hydrogels with enhanced mass transfer for transdermal drug delivery," Journal of Pharmaceutical Sciences, Vol. 85, No. 12, December 1996, PP. 1312-1316.

[7] N. Saha, N. Roy and P. Saha, "Allicin containing novel anti-microbial hydrogel," Proceedings Fifth International Conference on Polymer Modification, Degradation and Stabilization, Liege, Belgium, September 2008.

[8] N. Saha, A. Saarai, T. Kitano and P. Saha, "Seabuckthron oil incorporated medicated hydrogel based on gelatin sodium alginate," Proceedings SPE European Medical Polymers Conference, Belfast, United Kingdom, September 2008.

[9] A. Saarai, N. Saha, T. Kitano and P. Saha, "Natural resource based medicated hydrogel for health care," Proceedings Frontiers in Polymer Science, International Symposium celebrating the $50^{\text {th }}$ Anniversary of the journal Polymer, Mainz, Germany, June 2009.

[10] O. Z. Higa, S. O. Rogero, L. D. B. Machado, M. B. Mathor and A. B. Lugao, "Biocompatibility study for PVP wound dressing obtained in different conditions," Radiation Physics and Chemistry, Vol. 55, No. 5-6, August 1999, pp. 705-707. doi:10.1002/jbm.a.30308

[11] M. Sen and E. N. Avci, "Radiation synthesis of poly (N-vinyl-2-pyrrolidone)- $\kappa$-carrageenan hydrogels and their use in wound dressing applications. I. Preliminary laboratory tests," Journal of Biomedial Materials Research Part A, Vol. 74A, No. 2, August 2005, pp. 187-196.

[12] N. Roy, N. Saha, T. Kitano andP. Saha, "Novel hydrogels of PVP-CMC and their swelling effect on viscoelastic properties," Journal of Applied Polymer Science, Vol. 117, No. 3, August 2010, pp. 1703-1710.

[13] N. Roy, N. Saha, T. Kitano and P. Saha, "Development and characterization of novel medicated hydrogel wound dressing, Soft Materials, Vol. 8, No. 2, April 2010, pp. 130-148. doi:10.1080/15394451003756282

[14] N. Roy, N. Saha, P. Humpolicek and P. Saha, "Permeability and biocompatibility of novel medicated hydrogel wound dressings," Soft Materials, Vol. 8, No. 4, Nov 2010, pp. 338-357. doi:10.1080/1539445X.2010.502955

[15] G. D. Winter and J. T. Scales, "Effect of air drying and dressings on surface of a wound," Nature, Vol. 197, No.
4862, January 1963, pp. 91-92. doi:10.1038/197091b0

[16] X. Yang, K. Yang, S. Wu, X. Chen, F. Yu, J. Li, M. Ma and Z. Zhu, "Cytotoxicity and wound healing properties of PVA/ws-chitosan/glycerol hydrogels made by irradiation followed by freeze - thawing," Radiation Physics and Chemistry, Vol. 79, No. 5, May 2010, pp. 606-611. doi:10.1016/j.radphyschem.2009.12.017

[17] "Hydrogel burn and injury dressing", http://www.dae.gov.in/publ/betrlife/health/hydrogel.pdf

[18] L. Martineau and P. N. Shek, "Evaluation of a bi-layer wound dressing for burn care II. In vitro and in vivo bactericidal properties," Burns, Vol. 32, No. 2, March 2006, pp. 172-179. doi:10.1016/j.burns.2005.08.012

[19] K. Pal, A. K. Banthia and D. K.Majumdar, "Biomedical evaluation of polyvinyl alcohol - gelatine esterified hydrogel for wound dressing," Journal of Materials Science: Materials in Medicine, Vol. 18, No. 9, May 2007, pp. 1889-1894. doi:10.1007/s10856-007-3061-2

[20] J. Kopecek and J. Yang, "Review Hydrogels as smart biomaterials," Polymer International, Vol. 56, No. 9, September 2007, pp. 1078-1098. doi:10.1002/pi.2253

[21] P. Saha, N. Saha, N. Roy, "Hydrogel Wound Covering," Patent filed at Czech patent office (UPV CR). File number PV 2008-306 (2008).

[22] "Moisture content formula", http://www.tutorvista.com/math/moisture-content-formula

[23] T. Galya, V. Sedlarik, I. Kuritka, R. Novotny, J. Sedlarikova and P. Saha, "Antibacterial poly (vinyl alcohol) film containing silver nanoparticles: preparation and characterization," Journal of Applied Polymer Science, Vol. 110, No. 5, December 2008, pp. 3178-3185. doi:10.1002/mabi.200900131

[24] V. Rattanaruengsrikul, N. Pimpha and P. Supaphol, "Development of gelatine hydrogel pad as antibacterial wound dressings," Macromolecular Bioscience, Vol. 9, No. 10, October 2009, pp. 1004-1015.

[25] "The end zone: Measuring antimicrobial effectiveness with zones of inhibition", http://www.sciencebuddies.org/science-fair-projects/proje ct_ideas/MicroBio_p014.shtml

[26] K. R. Park and Y. C. Nho, "Synthesis of PVA/PVP hydrogels having two-layer by radiation and their physical properties," Radiation Physics and Chemistry, Vol. 67, No. 3-4, June 2003, pp. 361-365. doi:10.1016/S0969-806X(03)00067-7

[27] R. Barbucci, A. Magnani and M. Consumi, "Swelling Behavior of Carboxymethylcellulose Hydrogels in Relation to Cross-Linking, $\mathrm{pH}$, and Charge Density," Macromolecules, Vol. 33, No. 20, September 2000, pp. 7475-7480. doi:10.1021/ma0007029

[28] K. Pal, A. K. Banthia and D. K. Majumdar, "Preparation and characterization of polyvinyl alcohol - gelatin hydrogel membranes for biomedical applications," AAPS PharmSci Tech, Vol. 8, No. 1, March 2007, Article 21. doi:10.1208/pt080121 